EPJ Web of Conferences 59, 08001 (2013)

DOI: $10.1051 /$ epjconf/20135908001

(C) Owned by the authors, published by EDP Sciences, 2013

\title{
Development of a two arm, high energy, high power laser for plasma research in India
}

\author{
A.S. Joshi ${ }^{1, a}$, M.P. Kamath ${ }^{1}$, A.K. Sharma ${ }^{1}$, M.R. Raghuramaiah, \\ R.K. Patidar ${ }^{1}$, M.S. Ansari' ${ }^{2}$, N. Sreedhar ${ }^{2}$, R. Chandra ${ }^{2}$, C.P. Navathe ${ }^{2}$, \\ P.A. Naik ${ }^{1}$ and P.D. Gupta ${ }^{1}$ \\ 1 Laser Plasma Division, RRCAT, Indore 452013, India \\ 2 Laser Electronics Support Division, RRCAT, Indore 452013, India
}

\begin{abstract}
We report here work done towards development of a two arm, high energy, high power $\mathrm{Nd}$ :phosphate glass laser system. One arm of the laser is proposed to be operated in a long pulse $(\sim 1.5 \mathrm{~ns}$ pulse duration) with an energy of $\sim 400 \mathrm{~J}$. Presently, this arm of the laser is operating at energy of $\sim 100 \mathrm{~J}$ after a disc amplifier that amplifies the laser beam of diameter $\sim 94 \mathrm{~mm}$. After the addition of two more disc amplifiers which are nearing completion, the laser beam will have energy of $\sim 400 \mathrm{~J}$, with a beam diameter $140 \mathrm{~mm}$, at an intensity of $\sim 2 \mathrm{GW} / \mathrm{cm}^{2}$. This beam will be converted to its second harmonic using a $2 \times 2$ array KDP crystals. The second arm, under development, will operate with a hybrid amplification scheme using optical parametric chirped pulse amplification (OPCPA) and conventional amplification using the existing Nd:glass amplifiers to produce $50 \mathrm{TW}, 25 \mathrm{~J}, 500 \mathrm{fs}$ pulse. A tiled triangular pulse compressor is under development for compressing the stretched pulse.
\end{abstract}

\section{INTRODUCTION}

High energy, high power (HEHP) laser systems based on master oscillator power amplifier (MOPA) architecture [1-3] are widely used as pulse power drivers for high energy density physics studies like equation of state (EOS) of materials, generation of intense thermal x-ray radiation etc. A majority of the solid state HEHP laser chains use Nd:phosphate glass laser rods/discs as the active medium and xenon flash lamps as pump source [4]. HEHP systems are multi-arm lasers and are seeded with pulses of duration ranging typically from a few 100 ps to few ns from the master oscillator and deliver laser energy from $100 \mathrm{~J}$ to few $\mathrm{kJ}$ from each arm. In this paper, we will describe the development of two-arm Nd:phosphate glass laser system based on MOPA architecture that is being developed at Laser Plasma Division, RRCAT, Indore, India. The system is designed to deliver laser energy of $400 \mathrm{~J}$ over the pulse duration of $1.5 \mathrm{~ns}$ in one arm. The other arm will deliver laser pulse of $25 \mathrm{~J}$ of energy in $500 \mathrm{fs}(50 \mathrm{TW})$. This arm will have a hybrid amplification scheme using optical parametric chirped pulse amplifier (OPCPA) based chirped pulse amplification followed by amplification in Nd:glass amplifiers. The main aim of this project is a) to develop and use indigenously developed optics like laser glass rods and discs in the laser system, b) to develop the technology of OPCPA and the tiled grating pulse compressor, and c) in future, efforts will be made to amplify the long and short pulse with a low jitter and acceptable synchronization so that long and short pulses can be used for plasma experiments simultaneously.

\footnotetext{
ae-mail: asjoshi@rrcat.gov.in
}

This is an Open Access article distributed under the terms of the Creative Commons Attribution License 2.0, which permits unrestricted use, distribution, and reproduction in any medium, provided the original work is properly cited. 


\section{EPJ Web of Conferences}

\section{DESCRIPTION OF THE LASER SYSTEM}

The schematic diagram showing HEHP laser system is shown in Figure 1. The laser has two master oscillators. The oscillator for long pulse operations is SBS pulse compressed laser oscillator (SL-223: $\mathrm{M} / \mathrm{s}$ Ekspla, Lithuania) that delivers variable pulse width $(0.5-1.5 \mathrm{~ns})$ laser pulses with a contrast of better than $10^{4}$ and energy of $\sim 25 \mathrm{~mJ}$. The other oscillator generates seed laser pulses for the OPCPA. It is a commercial Kerr lens mode-locked (KLM) Ti:sapphire oscillator (Model: Chameleon Ultra II, $\mathrm{M} / \mathrm{s}$ Coherent Inc.), giving $100 \mathrm{fs}$ duration laser pulses (central wavelength: $1054 \mathrm{~nm}$ ) at $80 \mathrm{MHz}$ pulse repetition rate. The oscillator average power is $\sim 480 \mathrm{~mW}$ and the spectral bandwidth $(\Delta \lambda)$ of laser pulses is $\sim 11 \mathrm{~nm}$. The laser system has seven common laser glass rod amplifiers (named A-1 to A-7), after which there are two arms (referred to as arm-A and arm-B) each with three rod amplifiers (A-8-A-10) and three disc amplifiers (A-11 to A-13). Table 1 shows the details of the amplifiers in arm-A. It gives the typical operational energy and the intensity after each stage of the amplifier. It also gives the details of the electrical pumping through the xenon flash lamps; number of flash lamps used in an amplifier, their arc lengths, and bore diameters. The system is pumped by 198 flash lamps of various arc lengths as shown in Table 1, which in turn, are pumped by capacitor banks of total energy $\sim 700 \mathrm{~kJ}$, using bi-polar supplies [5]. The laser system is controlled by a microprocessor based electronic control system which is modular and is designed to control charging of capacitor banks of the amplifiers and firing them through the respective flash lamps at appropriate delays. This data is stored in the module by the microprocessor. Each bi-polar amplifier supply requires two control modules, one for the positive bank and another one for the negative bank [5]. The total system has around 40 such modules. This microprocessor is connected to a personal computer through a serial bus. For the user, a graphic user interface is provided to facilitate data entry and control operation. The control circuit and the power supply are isolated from each other by an isolation amplifier and an opto-coupler. The laser is sequentially relayed and spatially filtered laser system to control the degradation of spatial beam quality of the laser [6] as can be seen from Figure 1, which shows the layout of the laser system. The laser beam has a net accumulated nonlinear phase (B integral) contribution of $2 \mathrm{~Np}$ or less. The laser is presently available for the experiments with energy of $\sim 100 \mathrm{~J}$ with pulse duration of $\sim 1 \mathrm{~ns}$.

A schematic diagram of the 50 TW hybrid OPCPA [7] arm of the laser that is in advanced stage of development is given in the Figure 2. The pulses from the Ti:sapphire oscillator was stretched in double/four pass pulse stretcher giving $\sim 650 \mathrm{ps} / 1.3 \mathrm{~ns}$ pulses respectively. Both, the signal and pump laser pulses, were derived from the pulse stretcher after double and four passes in the pulse stretcher respectively [8]. The $1.3 \mathrm{~ns}$ pump pulse was amplified in a regenerative amplifier and two double pass amplifiers of 15 and $25 \mathrm{~mm}$ dia. as shown in Figure 2 and was converted to its second harmonic with $25 \%$ efficiency to pump the three Type I BBO crystals $(15 \mathrm{~mm} \times 15 \mathrm{~mm} \times 15 \mathrm{~mm})$ used as OPA. They were housed in an oven to maintain stable thermal environment. $20 \%$ energy of the pump beam was used to pump the first OPA stage and the remaining energy was used to pump remaining two stages of the OPA. Temporal overlap between the signal and the pump pulses was achieved using a commercial delay generator. Home-made electronic circuits were used to derive the electrical triggers for the flash lamps and various electro-optic devices controlling laser beam polarization. The variation of the OPA output pulse energy with the pump pulse energy after three OPA stages is shown in Figure 3. The theoretical curve based on saturated pulse amplification for the OPA is also given in Figure 3. A maximum gain of $\sim 8 \times 10^{5}$ was achieved in the three stage OPAs, resulting in an output energy of $300 \mu \mathrm{J}$, with an input signal energy of $400 \mathrm{pJ}$ at a pump pulse energy of $\sim 52 \mathrm{~mJ}$. The incident laser pulses had a bandwidth of $\sim 11 \mathrm{~nm}$ and the spectral bandwidth after OPA was measured to be $\sim 10 \mathrm{~nm}$, at the maximum output. The OPA output will be amplified to $\sim 40 \mathrm{~J}-50 \mathrm{~J}$ level in arm-B and then compressed using a triangular pulse compressor [9]. A stretched pulse of $\sim 650 \mathrm{ps}$ was compressed using the tiled pulse compressor in cold condition to give a pulse of $\sim 200$ fs [9].

Up-gradation of the above laser system by incorporating disc amplifiers for amplification of laser beam of diameters 115 and $140 \mathrm{~mm}$ is in progress. A $2 \times 2$ array of KDP crystals, each with a size of 


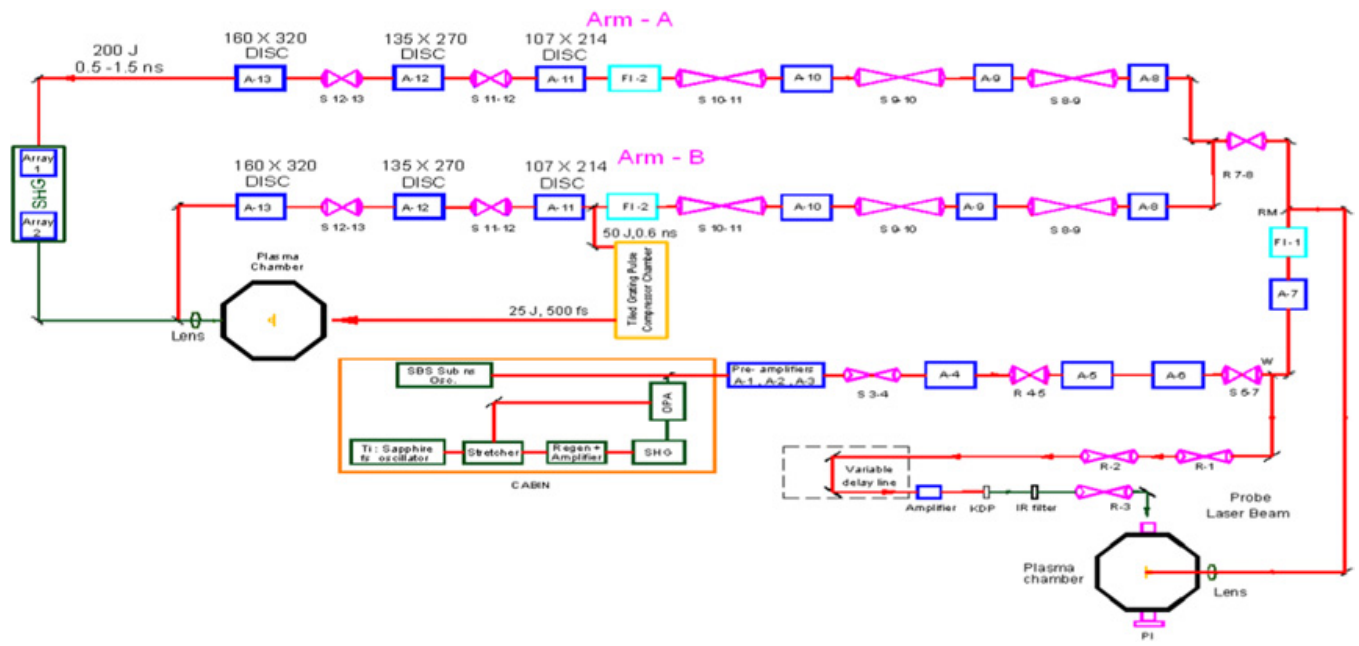

Figure 1. Schematic diagram of the high power laser chain.

Table 1. Data of amplifiers of the high power laser.

\begin{tabular}{|c|c|c|c|c|c|c|}
\hline No. & $\begin{array}{c}\text { Name of amplifier, } \\
(\text { Dia. mm for rods) } \\
(\mathrm{L} \mathrm{mm} \mathrm{x} \mathrm{B} \mathrm{mm)} \\
\text { for disc amplifiers }\end{array}$ & $\begin{array}{c}\text { No. of flash lamps } \\
\text { length }(\mathrm{mm}) \mathrm{x} \\
\text { bore dia }(\mathrm{mm})\end{array}$ & $\begin{array}{c}\text { Electrical } \\
\text { energy } \\
(\mathrm{kJ})\end{array}$ & Gain & $\begin{array}{c}\text { Operating } \\
\text { Energy }(\mathrm{J})\end{array}$ & $\begin{array}{c}\text { Operating } \\
\text { Intensity } \\
\text { GW/cm }\end{array}$ \\
\hline 1 & $\mathrm{~A}_{1}(10)$ & $4(150 \times 13)$ & 3.6 & 6 & 0.060 & 0.79 \\
\hline 2 & $\mathrm{~A}_{2}(10)$ & $4(150 \times 13)$ & 3.6 & 6 & 0.36 & 0.477 \\
\hline 3 & $\mathrm{~A}_{3}(15)$ & $4(180 \times 13)$ & 3.6 & 6 & 1.08 & 0.636 \\
\hline 4 & $\mathrm{~A}_{4}(25)$ & $6(280 \times 16)$ & 11.02 & 4 & 4 & 0.700 \\
\hline 5 & $\mathrm{~A}_{5}(50)$ & $10(280 \times 16)$ & 18.37 & 3.5 & 8 & 0.321 \\
\hline 6 & $\mathrm{~A}_{6}(50)$ & $10(280 \times 16)$ & 18.37 & 3.5 & 16 & 0.321 \\
\hline 7 & $\mathrm{~A}_{7}(65)$ & $12(280 \times 16)$ & 22.05 & 2 & 32 & 0.700 \\
\hline 8 & $\mathrm{~A}_{8}(65)$ & $16(280 \times 16)$ & 29.4 & 2 & 32 & 0.706 \\
\hline 9 & $\mathrm{~A}_{9}(65)$ & $16(280 \times 16)$ & 29.4 & 2 & 57.6 & 1.25 \\
\hline 10 & $\mathrm{~A}_{10}(80)$ & $20(280 \times 16)$ & 36.72 & 2 & 90 & 1.47 \\
\hline 11 & $\mathrm{~A}_{11}(107 \times 214)$ & $10(600 \times 16)$ & 36.72 & 1.4 & 124 & 1.05 \\
\hline 12 & $\mathrm{~A}_{12}(135 \times 270)$ & $6(750 \times 19)$ & 31.74 & & & \\
\hline 13 & $\mathrm{~A}_{13}(160 \times 320)$ & $6(900 \times 19)$ & 31.74 & & & \\
\hline
\end{tabular}

$75 \mathrm{~mm} \times 75 \mathrm{~mm}$, is under testing for conversion of the HEHP beam to its second harmonic in quadrature geometry.

\section{SUMMARY}

A two arm high power laser is under development in Raja Ramanna Centre for Advanced Technology, India. The laser is presently operational up to a disc amplifier (A-11) for amplification of $\sim 94 \mathrm{~mm}$ diameter laser beam. Two more disc amplifiers for amplification of 115 and $140 \mathrm{~mm}$ diameter beam (A-12, A-13) are under testing and will be coupled to the laser chain. A quadrature based, $2 \times 2$ array KDP second harmonic conversion system is also under development and will be shortly used in the 


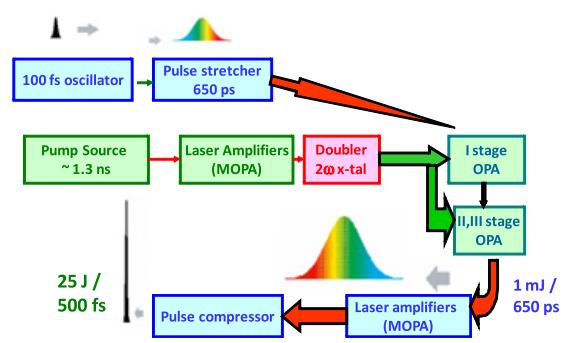

Figure 2. Schematic diagram of the 50TW hybrid OPCPA laser system.

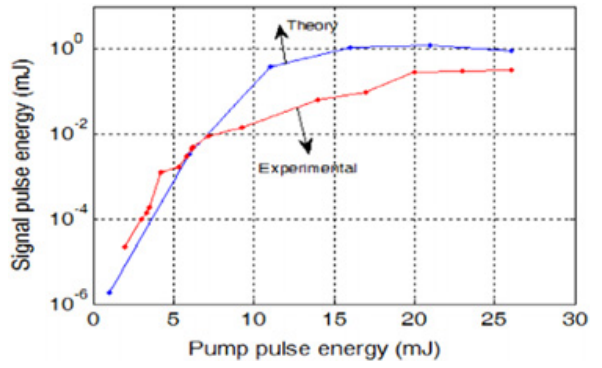

Figure 3. OPA Gain variation with input pump energy.

system. The control and the power supplies of all the amplifier stages of the laser system are ready. The laser output from the OPCPA based amplifier front end of the system will be fed into the Nd:glass amplifiers shortly. A triangular tiled grating pulse compressor has been assembled and coupled to the laser to deliver compressed pulses of $500 \mathrm{fs}$. Efforts will be made to operate the short pulse (Ti:sapphire) and long pulse (SBS pulse compressed) oscillators with minimal jitter so that both the pulses can be simultaneously used for the plasma experiments.

\section{References}

[1] T. R. Boehly, D. L. Brown , R. S. Craxton , R. L. Keck , J. P. Knauer, J. H. Kelly, T. J. Kessler, S. A. Kupman, S. J. Loucks, S.A. Letzring, F. J. Marshall, R. L. McCory, S. F. B. Morse, W. Seka, J. M. Soures, and C. P. Verdon, Opt. Commun. 133, 495 (1987)

[2] E. M. Campbell, J. T. Hunt, E. S. Bliss, D. R. Speck, and R. P. Drake, Rev. Sci. Instrum. 57, 2101 (1986)

[3] A. S. Joshi , C. P. Navathe , M. S. Ansari, M. P. Kamath, S. Bandhyopadhyay, A. Upadhyay, A.P. Kulkarni, P.K. Tripathi, S.R. Patwa, J.D. Singh, N. Sreedhar, R. Chandra, M.N. Kumbhare, and H. C. Pant, Fusion Engineering and Design 44, 67 (1999)

[4] J. H. Campbell, T. I. Suratwala, J. Non-Cryst. Solids 263 and 264, 318 (2000)

[5] C. P. Navathe, M. S. Ansari, S Nigam, S. Nyapathi, B. Singh, and R. Chandra, IEEE Trans. Plasma Science 40, 1898 (2012)

[6] J. T. Hunt, J. A. Glaze, W. W. Simmons, and P. Renard, Appl. Opt. 17, 2053 (1978)

[7] A. Dubietis, G. Jonusauskas and A. Piskarskas, Opt. Commun. 88, 437 (1992)

[8] M. Raghuramaiah, R.K. Patidar, R.A. Joshi, P.A. Naik, and P.D. Gupta, Pramana J. Phys. 75, 869 (2010)

[9] A.K. Sharma, M. Raghuramaiah, R. K. Patidar, A. S. Joshi, P. A. Naik, and P. D. Gupta, Proc.of Chirped pulse Amplification Symposium, Laval University, Canada, Nov 17-21, 2010 